\title{
First genetic analysis in Tunisian familial adenomatous polyposis probands
}

\author{
KARIM BOUGATEF ${ }^{1}$, RAJA MARRAKCHI ${ }^{1}$, AMEL MOUSSA ${ }^{2}$, YANNICK BLONDEAU-LAHELY ${ }^{3}$, \\ TAWFIK NAJJAR ${ }^{2}$, FLORENCE COULET ${ }^{3}$, CHRYSTELLE COLAS ${ }^{3}$, FARHAT BEN AYED ${ }^{4}$, \\ AMEL BEN AMMAR ELGAAIED ${ }^{1}$ and FLORENT SOUBRIER ${ }^{3}$ \\ ${ }^{1}$ Laboratoire de Génétique, Immunologie et Pathologies Humaines, Faculté des Sciences de Tunis, \\ Campus Universitaire 2092, Tunisia; ${ }^{2}$ Service Gastroentérologie, Hôpital Charles Nicolle de Tunis, Boulevard 9 \\ Avril 1938, Tunis-1006, Tunisia; ${ }^{3}$ Laboratoire d'Oncogénétique et d'Angiogénétique Moléculaire, Groupe \\ Hospitalier Pitié-Salpêtrière, 47-83, boulevard de l'Hôpital, 75651 Paris Cedex 13, France; ${ }^{4}$ Service de \\ Chimiothérapie, Institut de Cancérologie Salah Azaïez, Boulevard 9 Avril 1938, Tunis-1006, Tunisia
}

Received March 5, 2007; Accepted June 5, 2007

\begin{abstract}
Familial adenomatous polyposis (FAP) is an autosomal dominant inherited disease characterized by the development of hundreds to thousands of adenomatous polyps in colon and rectum. The APC gene (adenomatous polyposis coli) is considered as the major mutated gene in FAP. It has been shown that biallelic germline mutations in the baseexcision-repair gene MYH can be responsible for a recessive inheritance of adenomatous polyposis (AP). This study is the first Tunisian genetic analysis on AP patients. Multiplex ligation-dependent probe amplification (MLPA) was used to screen the APC gene for large genomic rearrangements. The total APC and MYH exon sequences and exon-intron edges were sequenced in an effort to detect germline mutations, four were explored. Mutations were detected in four patients that fulfil the clinical criteria of AP. Three mutations were found in the APC gene, of which two were novel (c.1636_1639delAGTG and c.2514 G>T) and all gave rise to a truncated APC protein. The missense G382D mutation, already described in north and south European populations was found in the MYH gene at the homozygous state in the fourth patient with moderate AP. Our preliminary study provides a basis for implementation of genetic counselling for AP.
\end{abstract}

\section{Introduction}

Colorectal cancer (CRC) is the first cause of digestive cancer mortality in Tunisia. Familial adenomatous polyposis (FAP)

Correspondence to: Dr Karim Bougatef, Laboratoire de Génétique, Immunologie et Pathologies Humaines, Faculté des Sciences de Tunis, Campus Universitaire, Tunis 2092, Tunisia

E-mail: karim.bougatef@fst.rnu.tn

Key words: familial adenomatous polyposis, MAP, colorectal cancer, screening, Tunisia, genetic tests is one of the hereditary colorectal cancers, and represent $1 \%$ of all cases of CRC (1). FAP is an autosomal dominant disease that affects about $1 / 10000$ individuals. Symptoms of FAP include abdominal pain, diarrhoea, haematochezia and mucus discharge but many patients are also asymptomatic. Patients with FAP develop hundreds to thousands of colon and rectum polyps (2) that may transform to cancer before the age of 40 if left untreated (3). Extracolorectal manifestations of FAP are common and characterise the Gardner syndrome (4), they include adenomas, osteomas, desmoïd tumours and congenital hypertrophy of the retinal pigment epithelium (CHRPE) (5).

FAP is caused by mutations of the adenomatous polyposis coli (APC) gene located on human chromosome region 5q21-22 (6). The APC gene is composed of 15 exons, and germline mutations have been described on the whole APC gene, but $\sim 65 \%$ of mutations are located in exon 15 that occupies $>75 \%$ of the whole coding region (7). Mutations in codon 1309 and 1061 (deletion of $5 \mathrm{bp}$ ) accounts respectively for $10 \%$ and $5 \%$ of all mutations described $(7,8)$.

The APC gene encodes a $311-\mathrm{kDa}$ multi-domain protein (the APC protein) involved in the Wnt signalling pathway (the wingless signal transduction pathway) (9). This protein binds to $\beta$-catenin and regulates the proliferation, migration and differentiation of the normal colorectal epithelial cells (10), where it acts as a 'gatekeeper' to prevent development of tumors. It was shown that APC plays a role in apoptosis, cellular adhesion and chromosome segregation (11).

Sieber et al (12) described a form of adenomatous polyposis (AP) without mutation of the APC gene and characterized by a lower number of polyps, typically $<100$. This form of AP shows a recessive mode of inheritance in families. Patients carry biallelic germline mutations in the baseexcision-repair (BER) gene MYH (MutYH), which encodes the DNA repair enzyme MYH glycosylase that is involved in the BER pathway, and conferring the term MYH associated polyposis to this particular form of AP (13). MYH protein removes mismatches 8-oxoG:A that occurs in DNA, preventing fixation of $\mathrm{G} / \mathrm{T}$ transversions. In case of biallelic inactivation 
of the MYH gene, DNA mutations can accumulate and cancer may develop $(13,14)$.

In Tunisia, FAP patients are diagnosed on the basis of family history, clinical and histological profile, but no genetic tests are available. The aim of this first Tunisian genetic study of FAP, was to analyze the entire coding region of the APC and MYH gene in four unrelated FAP Tunisian patients. This study enables the clinical spectrum of FAP in Tunisian patients to be assigned to the APC and MYH genes.

\section{Materials and methods}

Patients. This study includes 4 probands from unrelated families who came for counselling at Charles Nicolle Hospital of Tunis, because of a family history of colorectal cancer or unbearable symptom. After confirmation of the FAP state by colonoscopy or by suggestive history of hereditary CRC of polyposis type, consent for the genetic study was obtained from patients, the pedigree of the family was made and $10 \mathrm{ml}$ of peripheral blood was drawn in EDTA from each patient.

DNA isolation. Genomic DNA was purified from peripheral blood leukocytes using the QIAamp DNA blood mini kit (Qiagen; Valencia, CA, USA) following the manufacturer's instructions. A NanoDrop (ND-1000) spectrophotometer (Wilmington, DE, USA) was used to quantify the DNA.

Multiplex ligation-dependent probe amplification (MLPA). The Salsa MLPA P043 APC probemix kit (MRC-Holland, The Netherlands) was used according to the manufacturer's instructions to screen large deletions/duplications of one or more exons of the APC gene. In addition to probes for each exon, this kit contains one probe for alternative APC exon $10 \mathrm{~A}$ and two probes for the wild-type sequences at mutation hot-spots 1061 and 1309, allowing direct detection of small deletions at these locations. Fragment analysis of multiplex PCR was carried out on the ABI-3730 DNA analyser (Applied Biosystems, Foster City, CA, USA), using GeneMapper software, version 4.0 (Applied Biosystems). Deletions or duplications of probe recognition sequences are detected by a $35-50 \%$ reduced or increased relative peak area of the amplification product of that probe (15).

PCR amplification. All APC and MYH exons were amplified by polymerase chain reaction (PCR) in a total volume of $30 \mu \mathrm{l}$

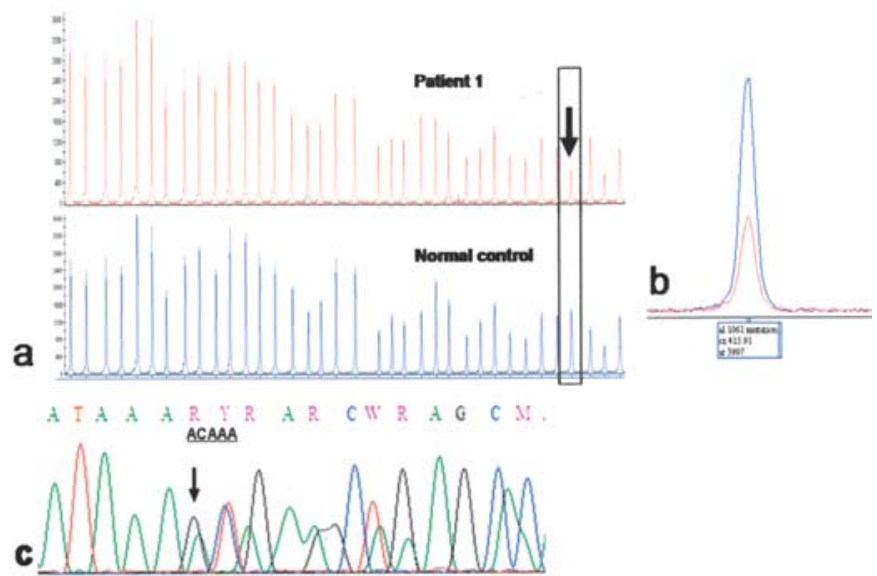

Figure 1. Detection of APC genomic deletion on patient 1 by multiplex ligation-dependent probe amplification (MLPA) and by sequencing. ( $a$ and $b$ ) APC normal control versus APC deletion in codon 1061 in patient 1 , screened by MLPA (the arrow shows the differences in the 1061 peak between control and patient 1), (c) Result of sequencing analyses: deletion of ACAAA in one allele in patient 1 (the arrow shows the position of the deletion).

of reaction mixture containing $100 \mathrm{ng}$ of genomic DNA of each sample, 1X PCR buffer, $2.5 \mathrm{mM} \mathrm{MgCl}_{2}, 10 \mathrm{mM} \mathrm{dNTP}$, $3.3 \mu \mathrm{M}$ of each primers designed with primer3 software (frodo.wi.mit. edu/cgi-bin/primer3/primer3_www.cgi) (Tables I and II) and $1 \mathrm{U}$ Ampli Taq Gold ${ }^{\circledR}$ DNA polymerase (Applied Biosystems). Amplification was performed on a GeneAmp PCR system 9700 (Applied Biosystems). The PCR products were analysed on an ethidium bromide stained agarose gel. Before sequencing, the positive reactions were cleaned up on a MultiScreen ${ }^{\mathrm{TM}}$ PCR 96-well plate (Millipore, Billerica, MA, USA).

Sequencing of genomic DNA. Sequencing reactions were carried out in forward and reverse orientations using the BigDye terminator v1.1 cycle sequencing kit (Applied Biosystems). The products of the sequencing reactions are cleaned up using the Sephadex ${ }^{\mathrm{TM}}$ G-50 (GE Healthcare, Life Sciences) in a MultiScreen ${ }^{\circledR}$-HV 96-well filter plate (Millipore), then run up on an ABI-3730 DNA sequencer. The resulting sequence data were analyzed with the SeqScape software, version 2.5 (Applied Biosystems) in comparison with the references sequences of the APC and

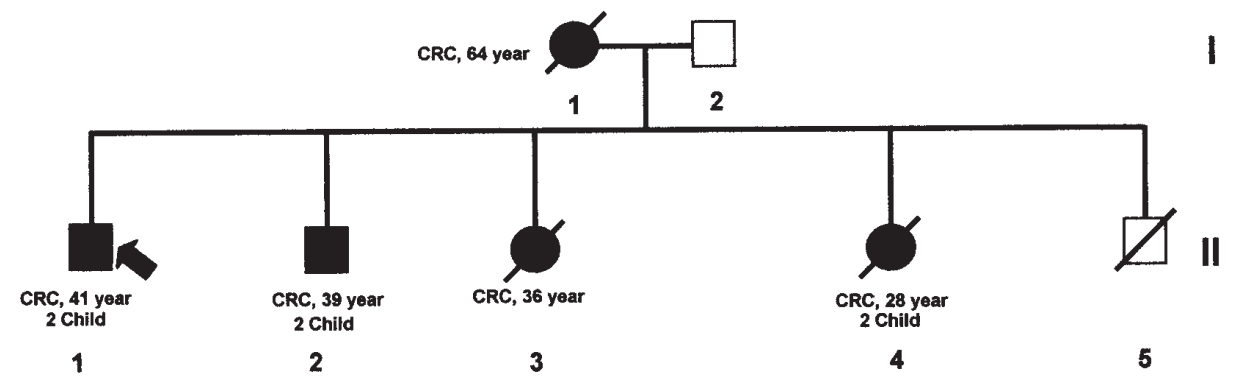

Figure 2. Family pedigree of patient 2. I-1, the patient's mother who had died of colorectal cancer at the age of 64; II-1, proband (patient 2); II-2, the patient's brother who had diagnosed with colorectal cancer at the age of 39; II-3, the patient's sister who had died of colorectal cancer at the age of 36; II-4, the patient's sister who had died of colorectal cancer at the age of 28 . The arrow shows patient 2. 
Table I. Primers and conditions for APC gene amplification by PCR.

\begin{tabular}{|c|c|c|c|c|}
\hline Exon & Forward primer & Reverse primer & $\operatorname{Tm}\left({ }^{\circ} \mathrm{C}\right)$ & $\begin{array}{l}\text { Fragment } \\
\text { length (bp) }\end{array}$ \\
\hline 1 & TTCTTTAAAAACAAGCAGCCACT & AAATGCTAACTTTTGCAAGAAAGA & 58 & 350 \\
\hline 2 & CAAGAAATACAGAATCATGTCTTGAA & ACCAACACCCAAATCGAGAG & 58 & 250 \\
\hline 3 & TGTTTTCAGTCATGTATATTTGTGG & TGGAGTACACAAGGCAATGTT & 58 & 350 \\
\hline 4 & TTGCTCTTCTGCAGTCTTTATT & TCAGGCCTAAAGTTGGGTAA & 48 & 300 \\
\hline 5 & TACAAGATATTGATACTTTTTTA & TGTAATTCATTTTATTCCTAATAGCTC & 48 & 210 \\
\hline 6 & TGATTTGACATAACCCTGAGC & GAACATCTATATTTCAATGGTGTCA & 58 & 270 \\
\hline 7 & AAGAAAGCCTACACCATTTTT & GATCATTCTTAGAACCATCTTGC & 50 & 220 \\
\hline 8 & TCATACAGACACTTCATTTGGA & CAAAATGCTGGGATTACAGG & 54 & 270 \\
\hline 9 & AGTCGTAATTTTGTTTCTAAACTC & GCTTTGAAACATGCACTACGAT & 58 & 440 \\
\hline 10 & ACAAAGCATTATGGTTTATGTTGA & TACCATGATTTAAAAATCCACCAG & 50 & 170 \\
\hline 11 & GATGATTGTCTTTTTCCTCTTGC & CTGAGCTATCTTAAGAAATACATG & 50 & 200 \\
\hline 12 & CCAAAAATAAAGCTTGGCTTC & GGTTGCAGTGAGCTGAGATT & 50 & 240 \\
\hline 13 & TTTCTATTCTTACTGCTAGCATT & ATACACAGGTAAGAAATTAGGA & 50 & 280 \\
\hline 14 & TAGATGACCCATATTCTGTTTC & CAATTAGGTCTTTTTGAGAGTA & 58 & 290 \\
\hline $15 \mathrm{AB}$ & GTTACTGCATACACATTGTGAC & TTGCTGGATGGTAGTTGCCT & 58 & 685 \\
\hline $15 \mathrm{CD}$ & ATTTGAATACTACAGTGTTACCC & TGTTTGGGTCTTGCCCATGTT & 48 & 724 \\
\hline $15 \mathrm{EF}$ & AGTCTTAAATATTCAGATGAGCAG & AGCTGATGACAAAGATGATAATG & 60 & 804 \\
\hline $15 \mathrm{GH}$ & AAGAAACAATACAGACTTATTGTG & TCCATCTGGAGTACTTTCTGTG & 50 & 770 \\
\hline $15 \mathrm{IJ}$ & AGTAAATGCTGCAGTTCAGAGG & TGAGCCTCATCTGTACTTCTGC & 60 & 725 \\
\hline $15 \mathrm{~K}$ & ATCTAACAATCGAATCCCCT & TTGTGGTATAGGTTTTACTGGTG & 54 & 350 \\
\hline $15 \mathrm{~L}$ & ACCCAACAAAAATCAGTTAGATG & GTGGCTGGTAACTTTAGCCTC & 60 & 400 \\
\hline $15 \mathrm{M}$ & ATGATGTTGACCTTTCCAGGG & ATTCTGTAACTTTTCATCAGTTGC & 58 & 230 \\
\hline $15 \mathrm{~N}$ & AAAGACATACCAGACAGAGGG & CTTTTTTGGCATTGCGGAGCT & 60 & 330 \\
\hline $15 \mathrm{O}$ & AAGATGACCTGTTGCAGGAATG & GAATCAGACGAAGCTTGTCTAGAT & 60 & 270 \\
\hline $15 \mathrm{P}$ & CCATAGTAAGTAGTTTACATCAAG & CTTAAGGGGTGGGCCTTTT & 54 & 230 \\
\hline 15Q & CAGCCCCTTCAAGCAAACATG & GAGGACTTATTCCATTTCTACC & 60 & 360 \\
\hline $15 \mathrm{R}$ & CAGTCTCCTGGCCGAAACTC & GTTGACTGGCGTACTAATACAG & 60 & 350 \\
\hline $15 \mathrm{~S}$ & TGGTAATGGAGCCAATAAAAAGG & TGGGAGTTTTCGCCATCCAC & 58 & 300 \\
\hline $15 \mathrm{~T}$ & TGTCTCTATCCACACATTCGTC & ATGTTTTTCATCCTCACTTTTTGC & 58 & 280 \\
\hline $15 \mathrm{U}$ & TTCCTCGAGTAAGCACTTGGA & TTGAATCTTTAATGTTTGGATTTGC & 58 & 420 \\
\hline $15 \mathrm{~V}$ & TCTCCCACAGGTAATACTCCC & GCTAGAACTGAATGGGGTACG & 60 & 260 \\
\hline $15 \mathrm{~W}$ & CAGGACAAAATAATCCTGTCCC & ATTTTCTTAGTTTCATTCTTCCTC & 50 & 330 \\
\hline
\end{tabular}

MYH gene (Accession number respectively; NM000038, NM_012222) downloaded from the NCBI Genebank website (http://www.ncbi.nlm. nih.gov/). All mutations were verified in both forward and reverse directions using PCR products from independent reactions.

\section{Results}

In this study, the genetic analysis of FAP was carried out on four unrelated Tunisian patients. Their age ranged between 13 and 43 years ( 3 males, 1 female). All displayed colorectal polyps, three had colon cancer and one underwent total colectomy. No extracolorectal manifestations were found in any of the patients. Our approach was to screen the APC gene by the MLPA technique to detect the possible presence of large genomic rearrangement (deletion, duplication) and to sequence the exons and intron-exon boundaries of the APC and MYH genes.

Patient 1 is a 35-year-old woman who presented with classical symptoms of FAP (>1000 polyps) and was diagnosed with colon cancer. MLPA analysis (Fig. 1a and b) revealed a known heterozygous mutation $(7,11)$ on codon 1061 of exon 15 in the APC gene (c.3183_3187 delACAAA). This 5-bp deletion introduces a frame-shift mutation and a stop codon (p.Lys1061fsX1062) and was confirmed by sequencing (Fig. 1c). Patient 2 is a 41 -year-old man that had contacted the Charles Nicolle Hospital of Tunis because of his concern of a family history of colon cancer. His mother and his two sisters had died of CRC. His brother was also affected with CRC (Fig. 2). On colonoscopy, he was found to 
Table II. Primers and conditions for MYH gene amplification by PCR.

\begin{tabular}{lcccc}
\hline Exon & Forward primer & Reverse primer & Tm $\left({ }^{\circ} \mathrm{C}\right)$ & $\begin{array}{r}\text { Fragment } \\
\text { length }(\mathrm{bp})\end{array}$ \\
\hline 1 & CTTCCCCTCTCCCAGAGC & GACCCGGGACGTCTGAAC & 60 & 254 \\
2 & CAAGAGTAAACCCGTGAGCA & AGCCTGAATCTGCCTTCAT & 59 & 329 \\
3 & AAGCCCTAAGTGGGAGCATA & CAGATGAGGAGTTAGGGTGGA & 55 & 323 \\
4 & ACCATGGAGAAGACGGGTAG & ATGAGGACACTGCTGACCTG & 62 & 239 \\
5 & CTGAGAGCAGGGCCACTT & GGGTGAAGGTGGTAGAGGAA & 60 & 276 \\
6 & GGTCAGACCCCAGATGAGAG & CCCGTCAGTCCCTCTATTGT & 63 & 200 \\
$7-8$ & GGACTGACGGGTGATCTCTT & CAGAGGGGCCAAAGAGTTAG & 62 & 382 \\
9 & CATTGCCTCTATCGCCTTTG & GAAGGGAACACTGCTGTGAA & 60 & 282 \\
$10-11$ & AGGATGTTGGGGTAACAAGG & AGAACTGGAATGGGGCTTCT & 60 & 432 \\
12 & TCTCTAGGTTGGCCCCTAAA & CGATTCCCTCCATTCTCTCT & 60 & 308 \\
13 & GGCAGTGGCATGAGTAACAA & CCTCAAAAGCCAACATCCTT & 59 & 260 \\
14 & CACCTTGGGGAGGTAAGTGA & GGAAACACAAGGAAGTACAACAAA & 57 & 291 \\
15 & GGACATGAAGTTAAGGGCAGA & AACTATTCCTCCCTCCAGTGAA & 51 & 250 \\
16 & CTACAAGGCCTCCCTCCTTC & ACATAGCGAGACCCCCATCT & 63 & 269 \\
\hline
\end{tabular}

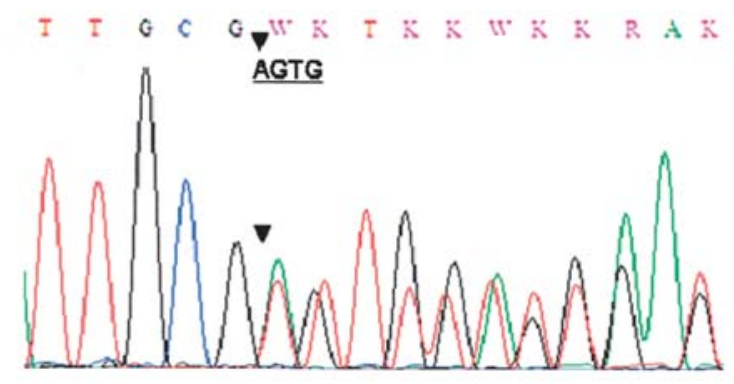

Figure 3. Detection of APC genomic deletion on patient 2 by sequencing. Deletion of AGTG in one allele at codon 546 (the arrows show the position of the deletion).
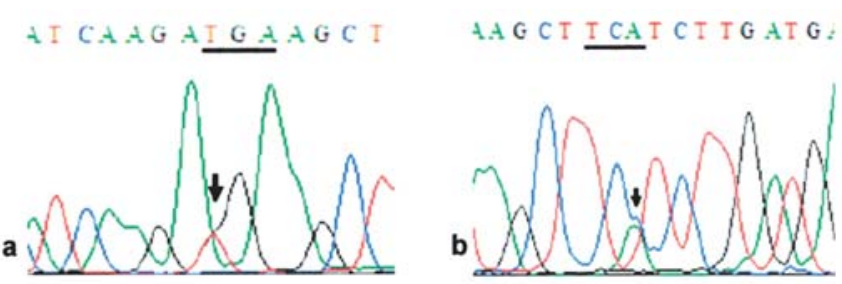

Figure 4. Detection of APC genomic transversion on patient 3 by sequencing. $\mathrm{G}$ to $\mathrm{T}$ transversion at the codon $839(\mathrm{GGA} \rightarrow \mathrm{TGA})$, resulting in a stop codon. (a) Forward sequence, (b) Reverse sequence (the arrows show the position of the substitution).

have a sigmoid adenocarcinoma and hundreds of polyps spread in the transverse and ascendant colon. He underwent a total colectomy. A heterozygous germline mutation was diagnosed for this patient in exon 13 of the APC gene by sequencing (Fig. 3). There was a frame shift mutation at codon 546 consisting of a four-nucleotide deletion (c.1636_1639delAGTG), resulting in a stop codon at position

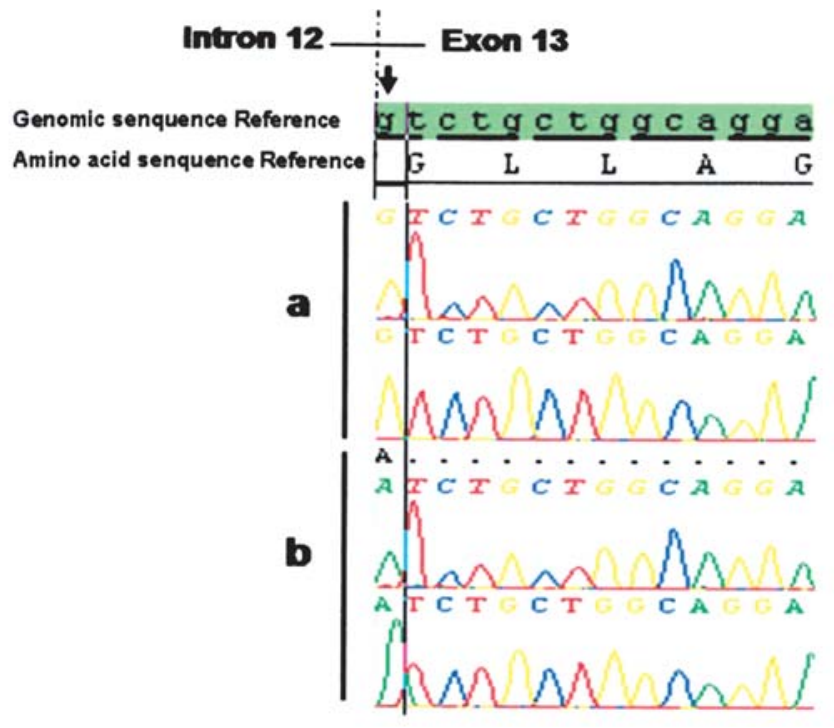

Figure 5. Alignment with the SeqScape software of two genomic MYH sequences showing a variation in the initial codon of exon 13. (a) Normal sequence with the guanine nucleotide at the 1145 position, (b) variant sequence of patient 4 with the homozygote adenosine nucleotide at the 1145 position.

547 (p.Ser546fs X 547). Since this mutation is absent of the APC database $(7,11,16)$ it appears that it is a novel germline mutation. Patient 3 is a 13-year-old child; his mother brought him to the hospital because his father and uncle had died of colorectal cancer at the fourth decade of their life and had presented major symptoms of FAP. The child refused colonoscopy. A novel germline mutation was detected and identified in the APC gene of this patient $(7,11,16)$. It is a heterozygous $\mathrm{G}$ to $\mathrm{T}$ transversion at codon 839 (GGA $\rightarrow$ TGA; c.2515 G>T), resulting in a stop codon (p.Gly839X) on one allele of the APC gene (Fig. 4). Patient 4 is a 43-yearold man who had an examination at the gastro-enterology 
clinic because of digestive pain; his colo-noscopy showed a limited number of polyps (eight tubular adenomas). No mutation was found in the APC gene either using the MLPA test or by the total sequencing of the APC gene. The analysis of the total MYH gene by sequencing showed an already characterized mutation $(17,18)$ in the first codon of exon 13 (Fig. 5), it is a G to A transition (c.1145 G>A; p.Gly382Asp) for which the patient is homozygous.

\section{Discussion}

This genetic study is the first mutation analysis of AP in Tunisia. We found a germline mutation in the four patients with clinical criteria of AP that were explored at the molecular level. We performed MLPA analysis and sequencing of the APC and MYH genes (exons and exons-intron boundaries) to detect mutations. We detected two novel germline mutations (Figs. 3 and 4) that are not mentioned in the APC data base $(7,11,16)$. These two mutations are located respectively at codon 546 and 839 , and result in premature stop codons. A third truncating mutation was detected in exon 15 of the APC gene at codon 1061 using the MLPA technique (Fig. 1a and b). This known mutation is recurrent and is also frequent in other populations $(7,11,16,19,20)$. The APC protein is an integral part of the wnt-signaling mechanism; it interacts with $\beta$-catenin and regulates its activity. The abolition of this interaction causes the accumulation of $\beta$-catenin protein in the cytoplasm of the colorectal cells thus promoting the generation of tumors $(21,22)$.

Investigation of the MYH gene showed for one patient (Fig. 5), a homozygous G382D germline missense mutation which has been reported as the most common deleterious mutation of the MYH gene in the Caucasian population $(12,18,14,23)$. In this case, the recessive phenotype of the disease is confirmed in this particular form of FAP linked to MYH mutation $(13,14)$. The effect of this MYH variant was studied by Al-Tassan et al (18) who showed that the mutated MYH protein expressed in Escherichia coli results in severe impairment of base excision repair. Subsequently, the biochemical function of the human G382 MYH protein was also shown to be severely compromised (24-26).

Our results are also important for genetic counselling and further efficient treatment and care of mutation carriers. Mutation identification can differentiate between the $50 \%$ risk of disease for progeny as observed for FAP with APC mutation, and the particular risk associated with consanguinity in the case of the recessive mode of disease inheritance for MAP. This study brings noteworthy new data concerning susceptibility to colon cancer due to FAP. It shows that even studying a restricted number of patients, we can confirm that the same genes are at play in the Tunisian population, including the APC and the more recently identified MYH gene. This has significant implications for genetic counselling in Tunisia, showing the importance of testing these two genes. Since consanguinity is a demographic characteristic in Tunisia, favouring the occurrence of recessive genetic diseases it is likely that the MYH gene is at play in a high proportion of AP. The mutation we found in the MYH gene is also a recurrent mutation in Caucasian population from north to south Europe, suggesting that it is a very ancient mutation (18).

A large molecular study of Tunisian patients should be undertaken, based on the presence of consanguinity and of early diagnosed colon tumors with clinical presentation compatible with MYH germline mutation in family members. Identification of MYH mutations would enable further genetic counselling and screening programs. Our study also confirms the heterogeneity of APC mutation by describing two new mutations of the APC gene in two of our patients. This clearly indicates that a large spectrum of mutations will be found in Tunisian patients.

From a public health point of view, and regarding economical constraints, our study clearly shows that stringent clinical criteria can yield a high proportion of positive molecular genetic results, which can further benefit rational screening and prevention.

\section{Acknowledgements}

We would like to thank all the members of 'Laboratoire d'Oncogénétique et d'Angiogénétique Moléculaire, Groupe Hospitalier Pitié-Salpêtrière, Paris, France' for their technical assistance.

\section{References}

1. Bisgaard ML, Fenger BS, Niebuhr E and Mohr J: Familial adenomatous polyposis (FAP): frequency, penetrance, and mutation rate. Hum Mutat 3: 121-125, 1994.

2. Bussey HJR: Familial polyposis coli. Family Studies, Histology, Differential Diagnosis, and Results of Treatment. The Hopkins University Press, Baltimore, 1975.

3. Bulow S: Familial polyposis coli. Dam Med Bull 34: 1-15, 1987.

4. Naylor EW and Gardner EJ: Adrenal adenomas in a patient with Gardner's syndrome. Clin Genet 20: 67-73, 1981.

5. Blair NP and Trempe CL: Hypertrophy of retinal pigment epithelium associated with Gardner's syndrome. Am J Ophthalmol 90: 661-667, 1980.

6. Bodmer WF, Bailey CJ, Bodmer J, Bussey HJR, Ellis A, Gorman P, Lucibello FC, Murday VA, Rider SH, Scambler P, Sheer D, Solomon E and Spurr NK: Localization of the gene for familial adenomatous polyposis on chromosome 5. Nature 328: 614-616, 1987.

7. Beroud C and Soussi T: APC gene: database of germline and somatic mutations in human tumors and cell lines. Nucleic Acids Res 24: 121-124, 1996.

8. Miyoshi Y, Ando H, Nagase H, Nishisho I, Horii A, Miki Y, Mori T, Utsunomiya J, Baba S, Petersen G, Hamilton SR, Kinzler KW, Vogelstein B and Nakamura Y: Germline mutations of the APC gene in 53 familial adenomatous polyposis patients. Proc Natl Acad Sci USA 89: 4452-4456, 1992.

9. Smith KJ, Johnson KA, Bryan TM, Hill DE, Markowitz S, Willson JK, Paraskeva C, Petersen GM, Hamilton SR, Vogelstein B, et al: The APC gene product in normal and tumor cells. Proc Natl Acad Sci USA 90: 2846-2850, 1993.

10. Bright-Thomas RM and Hargest R: APC, B-catenin and hTCF-4; an unholy trinity in the genesis of colorectal cancer. Eur J Surg Oncol 29: 107-117, 2003.

11. Fearnhead NS, Britton MP and Bodmer WF: The ABC of APC. Hum Mol Genet 10: 721-733, 2001.

12. Sieber OM, Lipton L, Crabtree M, Heinimann K, Fidalgo P, Phillips RK, Bisgaard ML, Orntoft TF, Aaltonen LA, Hodgson SV, Thomas HJ and Tomlinson IP: Multiple colorectal adenomas, classic adenomatous polyposis, and germ-line mutations in MYH. N Engl J Med 348: 791-799, 2003.

13. Boiteux S and Radicella JP: Base excision repair of 8-hydroxyguanine protects DNA from endogenous oxidative stress. Biochimie 81: 59-67, 1999. 
14. Halford SE, Rowan AJ, Lipton L, Sieber OM, Pack K, Thomas HJ, Hodgson SV, Bodmer WF and Tomlinson IP: Germline mutations but not somatic changes at the MYH locus contribute to the pathogenesis of unselected colorectal cancers. Am J Pathol 162: 1545-1548, 2003.

15. Sellner LN and Taylor GR: MLPA and MAPH: New techniques for detection of gene deletions. Hum Mutat 23: 413-419, 2004.

16. Soussi T: UMD APC mutation data base. Available at http://www.umd.necker.fr/, 2003.

17. Croitoru ME, Cleary SP, Di Nicola N, Manno M, Selander T, Aronson M, Redston M, Cotterchio M, Knight J, Gryfe R and Gallinger S: Association between biallelic and monoallelic germline MYH gene mutations and colorectal cancer risk. J Natl Cancer Inst 96: 1631-1634, 2004.

18. Al-Tassan N, Chmiel NH, Maynard J, Fleming N, Livinston AL, Williams GT, et al: inherited variants of MYH associated with somatic G:C $\rightarrow$ T:A mutations in colorectal tumors. Nat Genet 30: $227-232,2002$.

19. Guillem JG, Smith AJ, Culle J and Ruo L: Gastrointestinal polyposis syndromes. Curr Probl Surg 36: 425-435, 1999.

20. Cruz-Bustillo D, Villasana L, Llorente F, Casadesus D, Garcia E, Syrris P, Carter N, Guerra R and Llanio R: Preliminary results of the molecular diagnosis of familial adenomatous polyposis in Cuban families. Int J Colorectal Dis 17: 344-347, 2002.
21. Fodde R, Smits R and Clevers H: APC, signal transduction and genetic instability in colorectal cancer. Nat Rev 1: 55-67, 2001.

22. Lee S, Hong S, Shin SJ, et al: Paillary thyroid carcinoma associated with familial adenomatous polyposis: molecular analysis of pathogenesis in a family and review of the literature. Endocr J 51: 317-323, 2004.

23. Enholm S, Hienonen T, Suomalainen A, Lipton L, Tomlinson I, Karja V, et al: Proportion and phenotype of MYH-associated colorectal neoplasia in a population-based series of Finnish colorectal cancer patients. Am J Pathol 163: 827-832, 2003.

24. Chmiel NH, Livingston AL and David SS: Insight into the functional consequences of inherited variants of the hMYH adenine glycosylase associated with colorectal cancer: complication assays with hMYH variants and pre-steady-state kinetics of the corresponding mutated E. coli enzymes. J Mol Biol 327: 431-443, 2003.

25. Wang L, Baudhuin LM, Boardman LA, Steenblock KL, Petersen GM, et al: MYH mutations in patients with attenuated and classic polyposis and with young-onset colorectal cancer without polyposis. Gastroenterology 127: 9-16, 2004.

26. Kambara T, Whitehall V, Spring KJ, Barker MA, Arnold S, Wynter C, Matsubara N, et al: Role of inherited defects of MYH in the development of sporadic colorectal cancer. Genes Chromosomes Cancer 40: 1-9, 2004. 